\title{
Design Fiction: Anticipating Adoption
}

Paul Coulton and Joe Lindley

\section{Introduction}

When we submitted our 'work in progress' (WiP) paper Game of Drones [1], to 'CHI Play' in 2015 , we had no idea whether we would be derided or praised, as Game of Drones is a fictional account of a research project that had never actually happened. Whilst it may be surprising to some that such a paper passed review and was even accepted, it is important to understand the intent behind this paper was not subversive but rather to produce new knowledge [2]. The purpose of this project was dual: we wanted to explore a potential future use of drones for civic enforcement activities and also to progress a programme of research that aims to develop Design Fiction as a research method. In this tutorial we endeavour to highlight the enormous potential of Design Fiction by covering both facets: how the drones helped us develop a Design Fiction, and how Design Fiction helped us to highlight the wider issues relating to design of such a drone-based system.

The term 'drone' covers a broad range of unmanned aerial vehicles, but it is the proliferation of small quadcopters for personal use that has made them prominent in the public consciousness. Multi-rotor copters come with sophisticated flight controllers and onboard sensors which make them ever-easier to control. Their ease-of-use and relatively low cost have facilitated a huge range of controversial, sometimes amusing, and often innovative applications. For example, delivering drugs to prison inmates, a platform for espionage, flying cats and Halloween ghosts, a plethora of photographic applications, aerial light painting and competitive first-person view drone racing. Amazon's Prime Air, Facebook's internet drone, and a huge range of wildlife conservation drones are further examples of innovative commercial, corporate, and research uses of the same technologies. What these activities demonstrate is that while there is a high degree of 'interpretive flexibility' around drone technologies their full significance for society has not 'stabilised' as they have yet to be 'domesticated' and adopted into wide spread practices.

Bell \& Dourish highlighted that 'we' (technology researchers) have a penchant for suggesting that the actual adoption of the technologies we research is 'just out of reach' or 'around the corner', and that considering how this is achieved, or possible unexpected consequences, is left as someone else's problem [3]. Of course, researchers are not able to fully envisage the gamut of potential futures for emerging technologies but this doesn't mean we shouldn't try. Design Fiction is an approach that can help us to shift our gaze from the foreground of the present, to the horizon of the future, and then back again. Having glimpsed possible futures, we can be better prepared to consider the broader range of societal implications. Therefore, Design Fiction is not about predicting the future or creating utopian visions that promote emerging technologies, instead it provides a means to explore the societal, technological, and political nuances of possible futures so that we can better understand our present. Drone technology, along with Internet of Things, Smart Cities, and Autonomous Automobiles, are on the cusp of widespread adoption, they are at a peak of expectation, and hence examining proximate futures that plausibly extend from today's 
present is particularly relevant for these technologies. In the example used here, Game of Drones, we were interested in exploring a drone-based gamified civic enforcement system. We wanted to understand what the technological and legal challenges of such a system would be, and to ask whether such a system would be an acceptable proposition and to start a discourse that starts to unpack the ethical and societal questions relating to such a system's adoption.

\section{Design Fiction}

We have come to see Design Fiction as a world building exercise. By creating a series of speculative artefacts, the worlds that these artefacts inhabit spring into life. Given life, these fictional worlds provide a prototyping medium for the designed artefacts which define them, meanwhile the designs reciprocate by prototyping the world itself. We propose two metaphors for describing how the individual artefacts relate to the world. First, let's imagine a Design Fiction world as a distinct entity, one that we can see the overall shape of, but whose complex internal structure is hidden from view. What we can see, however, is a series 'entry points'. Each artefact that contributes to making up the Design Fiction acts as a metaphorical entry point to the fictional world as shown in figure 1. The second metaphor, is inspired by Charles and Ray Eames' film about relative size of things in the Universe, 'Powers of 10'. The film shows a number of frames of reference (literally drawn as squares in the film) which begin with a 1 meter squared section of an image that includes a couple sitting having a picnic, but then zooming out and increasing the visible area by one power of 10 every 10 seconds. Within 1 minute the image shows a view of Earth from outer space, 3 minutes later and the frame has zoomed out far enough to show the entire observable Universe. Although we are not suggesting adherence to the configuration '1 power of 10 per 10 seconds', the basic concept can be applied to Design Fiction worlds and the artefacts that create them. We can think of each individual artefact that constructs the world as a representation of that world, but at a different scale or 'power of 10' (see figure 1). Entry points at varying scales makes the artefacts that construct them, and design fiction worlds themselves, incredibly flexible. However, it is their situated character that makes Design Fiction such a powerful tool. The technique goes beyond just exploring the utility, usability or technical requirements for a specific technology but instead takes all of these factors into account, and at the same time places people at the heart of the scenarios, and does so with technologies or systems that are not currently in existence.

\section{But what about the drones?}

The world we built for Game of Drones was constructed using a variety of artefacts and then the whole 'world' was packaged into the submitted paper. The WiP paper format provided the opportunity to focus on individual elements of the system and write about how they fitted together, as opposed to needing to create believable results. The structure of the paper also allowed us to evaluate whether our imaginary world was plausible to the communities developing such technologies, rather than creating a vision that is clearly fictional and might easily be dismissed as science fiction. By adhering to a normal paper structure we rendered a 'future mundane', in which drones are an everyday part of the urban landscape. The paper describes a user trial of the Drone Enforcement System (DES) in which drones are used to provide enforcement services to local authorities. Specifically, it presents a 'gamified' system, which allowed retired members of the police and armed 
services to act as remote drone pilots helping to enforce by-laws relating to parking offences and dog fouling in a small UK city. The whole interaction takes place through a game-like interface and points are awarded to pilots for recording activity, and ultimately, catching other citizens infringing upon the rules. Alongside the paper we submitted a supporting video which comprised of real footage recorded from a drone but was composited with a game-like interface and claimed - fictionally - to show the system 'in the wild' (figure 2). In the following paragraphs we will discuss important elements that shaped the design of the system presented in the paper and video.

\section{Your user trial is illegal? Rewrite the law}

Within current UK legislation the adoption of commercial services, such as the one described in Game of Drones, would be unfeasible because of Civil Aviation Authority Protocol 658 (Article 167) which governs small unmanned surveillance aircraft of less than $7 \mathrm{Kg}$ in weight. In particular, the article stipulates that, currently, drones must not be flown within 50 meters of any person, structure or vehicle and when flown via a First Person View (FPV) camera on the drone, they are required to adopt a 'Buddy Box' system whereby another person maintains a line of site view and can take over control of the drone if required. Within the realms of our fictional world, we therefore had to consider a future in which this article no longer applied and thus superseded Article 167 with our own law that allows remote FPV flight for users in possession of a 'Drone Pilot Proficiency Certificate' within certain height and distance limitations. Although only part of our more comprehensive Design Fiction world, this change in legislation is a Design Fiction prototype in its own right, and was arguably a portend to the US Federal Aviation Administration's subsequent implementation of compulsory drone registration and mandatory certification for commercial pilots.

\section{Need to recharge your drone? Design a docking station}

A number of technical details about hardware are included in Game of Drones, most notably; a contemporarily available consumer model of a drone and camera (cited as being used in the trial), as well as a sketch of the docking station design (figure 3), photographs of signage (figure 4), and a diagram of the control device. While the drone hardware primarily played a supporting role in the Design Fiction, it allowed us to consider how current their current capabilities would impact upon the proposed system design. With this in mind the drone docking station was designed based on a real type of lamppost (figure 2 ) and would have been a necessary part of the infrastructure for this system due to the limited flight time of these battery powered vehicles. To further bolster the plausibility of the fiction we incorporated wireless charging technology (citing real research) into the docking stations and, because it would be necessary to make landing on the docking stations far simpler, we incorporated automatic landing beacons. We note that subsequent to writing the paper, Amazon were filed for patent US009387928 in July 2016 which describes a remarkably similar lamppost-based docking station for drones to the one we proposed.

\section{Drone-zone signs, or it didn't happen.}

A number of extra details were added about the trial, all of which were intended to be reminiscent of reality, but with twists for the Game of Drones world applied to them. These all add further to the Design Fiction's plausibility, but also act as cultural triggers to spark 
meaningful discussion. Most of us are familiar with parking or dog fouling notices, hence, we made real 'Drone Enforcement Zone' signs (figure 3) that utilize the official UK font for road signage. In addition, real GIS data was used to construct a map of the trial city, complete with enforcement zones (based upon existing city council maps of parking zones) and landing stations placed to facilitate coverage based on our flight distance restrictions and practical recharging. Programmatically enforced no-fly zones are mentioned for special areas such as the railway line to acknowledge current discourses about the potential hazards caused by drones. Finally, we claimed that the trial participants were ex-service (police and military) personnel, as they would likely have a diligent approach to enforcement. A note about financial rewards suggests that for this iteration of the system, users are not financially rewarded for recording more infringements, hinting at the possible ethical dilemmas of gamifying enforcement. It might seem that we went to a lot of trouble for such small details but we believe they add important texture to the fictional world, making it appear more plausible, and in doing so aim to stimulate more meaningful discourse. For instance, "the drone pilots are also encouraged to record any activity they consider 'unusual' to ascertain the use of drones has potential for crime prevention beyond enforcement activities" [1] could suggest to some visions of a 'big brother' style dystopia, while for others it may be reminiscent of Neighborhood Watch schemes. This ambiguity is deliberate and done with the aim of encouraging discussion about the desirability of such systems and their effect on society.

\section{Conclusions}

Game of Drones builds a fictional world in which drones are used as part of a gamified civic enforcement system. It does so convincingly enough that two out of three of the reviewers seemed to believe it was real despite our admission in the paper's conclusion that it was part of a Design Fiction, and therefore a 'fictional account' [2]. This level of acceptance, despite the evidence, is fascinating, not because of the misinterpretation but because it demonstrates how powerful technological visions of the future even to those shaping such futures and, in this particular case, that not only is the proposed drone-based enforcement system plausible, it also seems to be feasible. It could (and likely will) become reality. If this is the case, it is important to have wider discussions about the prospect now. Overall, we assert that the technique demonstrated in this tutorial, Design Fiction, is becoming an increasingly relevant approach for designers and researchers through which to explore the wider implications of adopting emerging technologies of all kinds, the Internet of Things, synthetic biology, nanotechnology, and - of course - drones.

\section{Acknowledgements}

This spotlight has been made possible through the support of the RCUK Cyber Security for the Internet of Things Research Hub PETRAS (petrashub.org) under EPSRC grant $\mathrm{EP} / \mathrm{N} 02334 \mathrm{X} / 1$.

\section{References}

[1] Joseph Lindley and Paul Coulton. 2015. Game of Drones. In Proceedings of the 2015

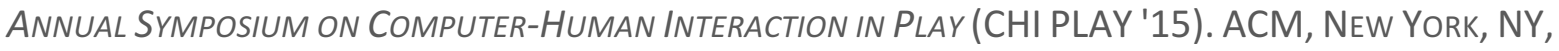
USA, 613-618. DOI: HTTP://DX.DOI.ORG/10.1145/2793107.2810300 
[2] Joseph Lindley and Paul Coulton. 2016. Pushing the Limits of Design Fiction: The Case For Fictional Research Papers. In Proceedings of the 2016 CHI Conference on Human Factors in COMPUTING SYSTEMS (CHI '16). ACM, NEW YORK, NY, USA, 4032-4043. DOI:

HTTP://DX.DOI.ORG/10.1145/2858036.2858446

[3] GeneVIeVe Bell and Paul Dourish. 2007. Yesterday's tomorrows: notes on ubiQuitous COMPUTING'S DOMINANT VISION. Personal UbiQUitous Comput. 11, 2 (January 2007), 133-143. DOI=HTTP://DX.DOI.ORG/10.1007/s00779-006-0071-X

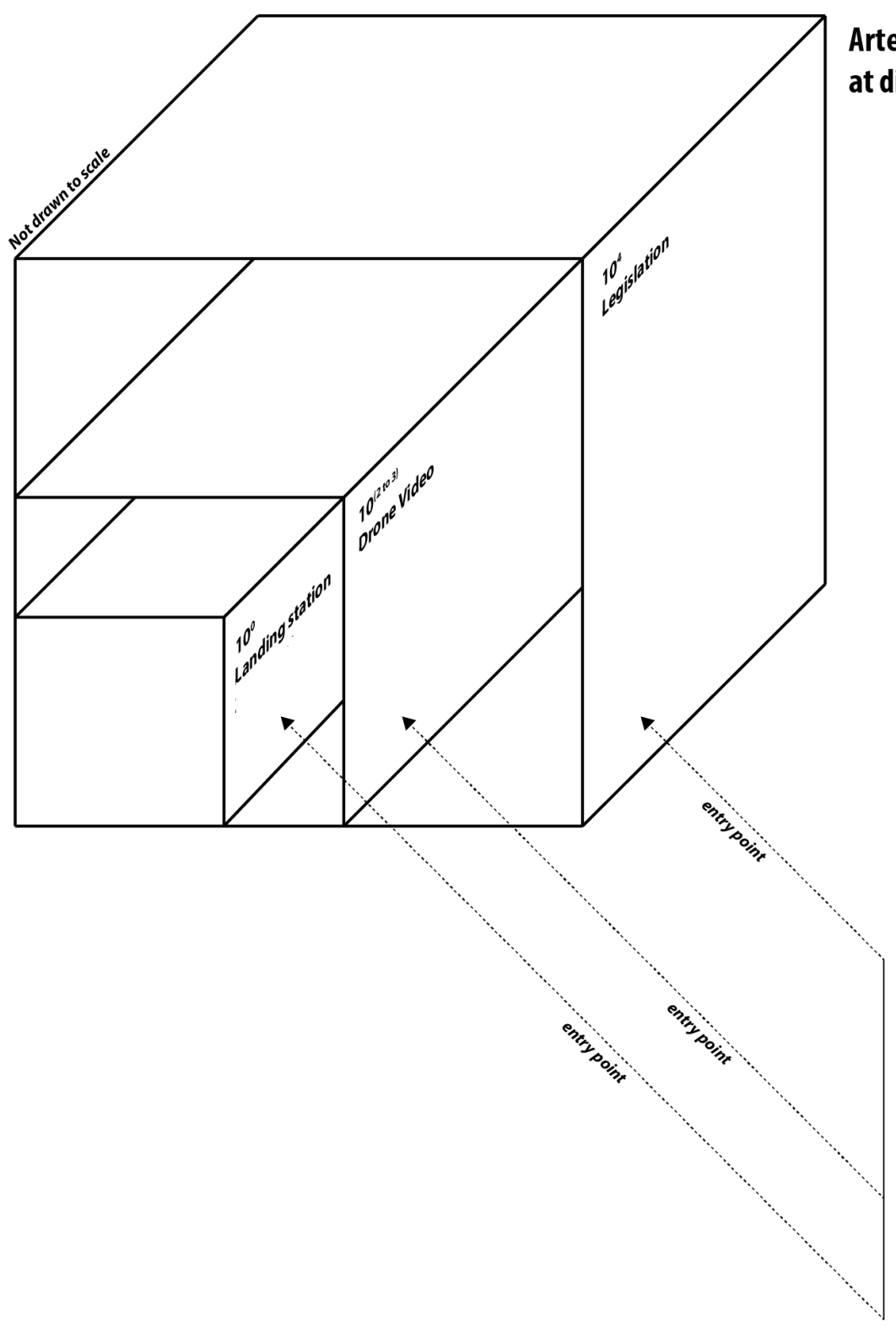

Each artefact represents an 'entry point' into the world

Figure 2. Design Fiction World Building 

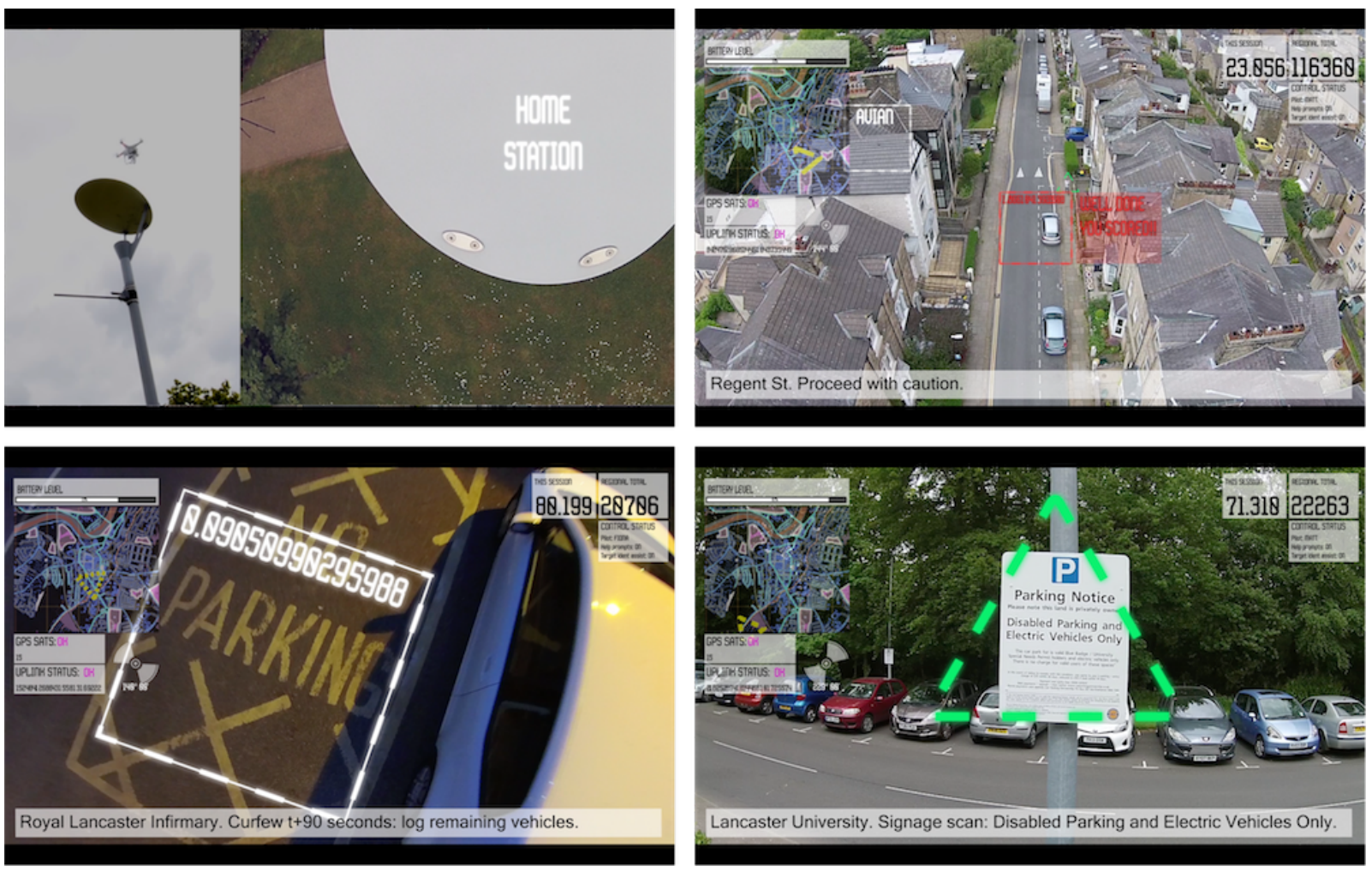

Figure 2. Screenshots from Game of Drones Video (https://youtu.be/6b_30d7yW2s)

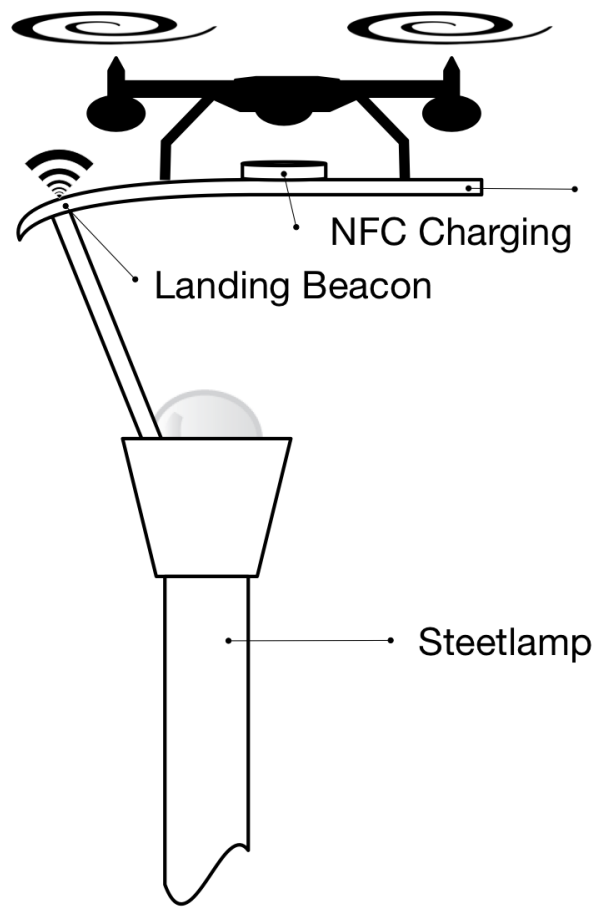

Reflector acts as drone base

Figure 3. Drone Docking Station 

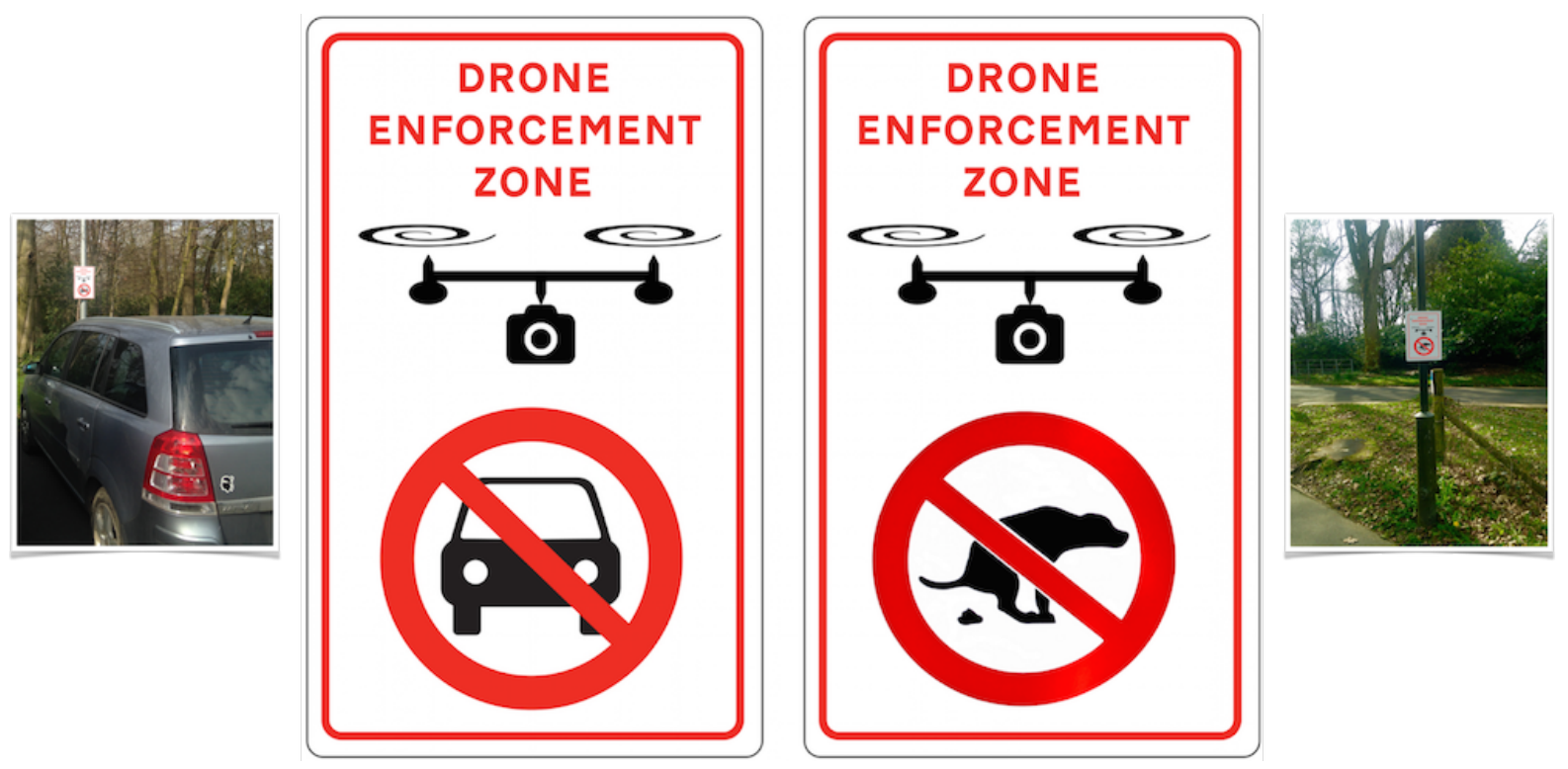

Figure 4. Drone Enforcement Zone Signage

Additional Image Game of Drones Logo

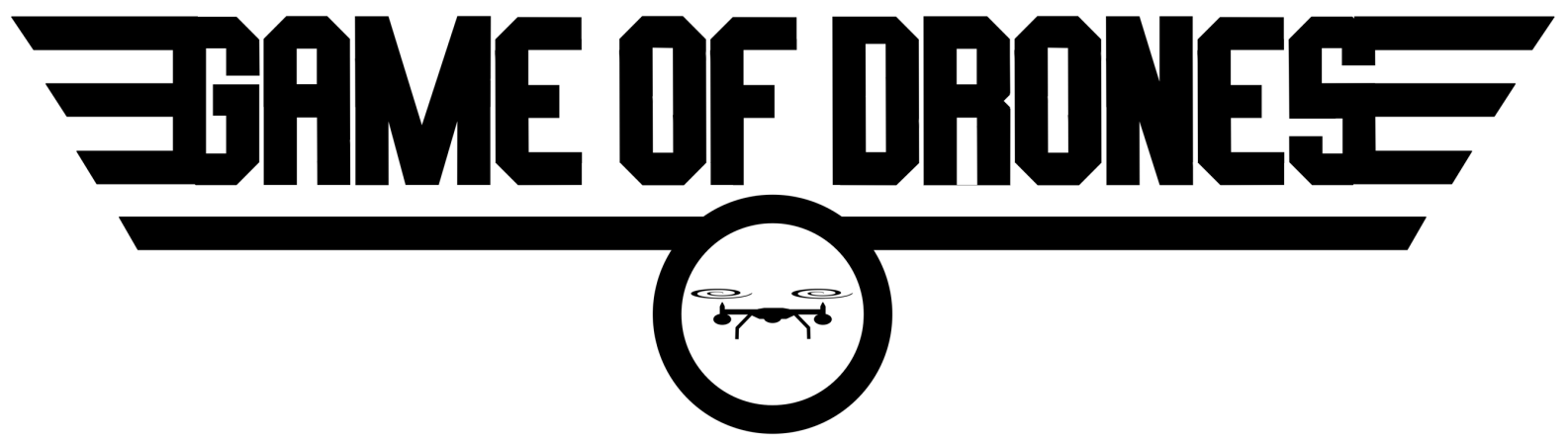

